\title{
Measurement and tuning of the chromatic dispersion of a silicon photonic wire around the half band gap spectral region
}

\author{
François Leo, ${ }^{1, *}$ Utsav Dave, ${ }^{1}$ Shahram Keyvaninia, ${ }^{1}$ Bart Kuyken, ${ }^{1}$ and Gunther Roelkens ${ }^{1}$ \\ ${ }^{1}$ Photonics Research Group - Center for nano- and biophotonics (NB-Photonics), \\ Sint-Pietersnieuwstraat 41, B-9000 Ghent, Belgium
}

compiled: October 24, 2013

\begin{abstract}
We demonstrate the measurement and tuning of second-to-fourth order dispersion of a silicon wire waveguide in a spectral region of low nonlinear losses. Using white light interferometry we extract the chromatic dispersion of our waveguide from $1950 \mathrm{~nm}$ to $2300 \mathrm{~nm}$. Moreover we demonstrate tuning of the zero dispersion wavelength over more than $100 \mathrm{~nm}$, pushing it to longer wavelength by partially underetching the waveguide.
\end{abstract}

OCIS codes: (130.4310) Integrated optics, Nonlinear; (260.2030) Physical optics, Dispersion

http://dx.doi.org/10.1364/XX.99.099999

Nonlinear silicon photonics is attracting a lot of attention because of its possible application to high speed signal processing and spectroscopy [1]. Due to the high confinement offered by the silicon-on-insulator (SOI) platform (as a result of the high index contrast between silicon and silica) and the intrinsically high nonlinear index of silicon, nonlinear parameters 5 orders of magnitude higher than standard optical fibers have been reported [2]. However, in order to fully benefit from such high nonlinearity, one should work above the half band gap spectral region i.e. $\lambda>2.2 \mu \mathrm{m}$, where two photon absorption based nonlinear losses significantly drop [3]. Recent results such as parametric amplification [4] and supercontinuum generation [5], have confirmed the large potential of this spectral region. Most nonlinear processes require good tailoring of the group velocity dispersion of the guided mode. Anomalous dispersion is necessary to achieve phase matching for parametric amplification or to reach solitonic propagation [6]. Strong modal confinement is necessary to achieve such anomalous regime as the material dispersion of silicon is strongly normal [7]. Here we focus on photonic wire waveguides with a standard silicon waveguide layer thickness of $220 \mathrm{~nm}$, easily available through cost-sharing platforms such as epixfab [8]. Moreover it is in such waveguides that a record nonlinear parameter of $150(\mathrm{Wm})^{-1}$ was recently reported in the half band-gap spectral region [9]. Full-vectorial mode solver simulations show that the region of anomalous dispersion is highly sensitive to silicon waveguide layer thickness and waveguide width. Optimal operation for $220 \mathrm{~nm}$ silicon thickness is found for a waveguide width of $900 \mathrm{~nm}$, with

\footnotetext{
* Corresponding author: francois.leo@intec.ugent.be
}

an anomalous region extending up to $2400 \mathrm{~nm}$ [5] However, waveguide widths and especially silicon waveguide layer thickness can vary within a wafer and from wafer to wafer which can lead to very different dispersion profiles than expected, which is detrimental for nonlinear applications. In this paper we adapt a well known interferometric method used for fibers [10] to measure the dispersion in these wire waveguides. Previous measurements of the dispersion of silicon waveguides, using either whitelight interferometry $[11,12]$ or integrated Mach-Zehnder interferometers [13] were limited to a narrow telecom band. Here we show that such a technique can be easily adapted to any spectral region where broadband sources and detectors are available. We demonstrate measurements of second to fourth order dispersion from $1950 \mathrm{~nm}$ up to $2300 \mathrm{~nm}$, a wavelength range of particular interest for nonlinear optics as discussed above. Moreover, we propose underetching as a technique to tune the anomalous region to longer wavelength. Dispersion tailoring and tuning of nanophotonic waveguides for nonlinear optics applications has been intensely studied in recent years. Tuning was performed by depositing dielectric overlayers through chemical vapour deposition [14] or atomic layer deposition [15]. However such techniques decrease the vertical confinement which dont allow tuning the anomalous region to longer wavelength in this case. Here we use underetching, i.e. removing the silicon dioxide by wet etching, in order to increase the vertical confinement of the optical mode and shift the zero dispersion wavelength (ZDW) to longer wavelengths. There are two other advantages of this technique compared to the deposition of dielectric overlayers : firstly, the possibility to very precisely control the etch rate and hence the undercut (a rate of $1 \mathrm{~nm} / \mathrm{min}$ was achieved); secondly, the modal confinement is increased, thereby in- 


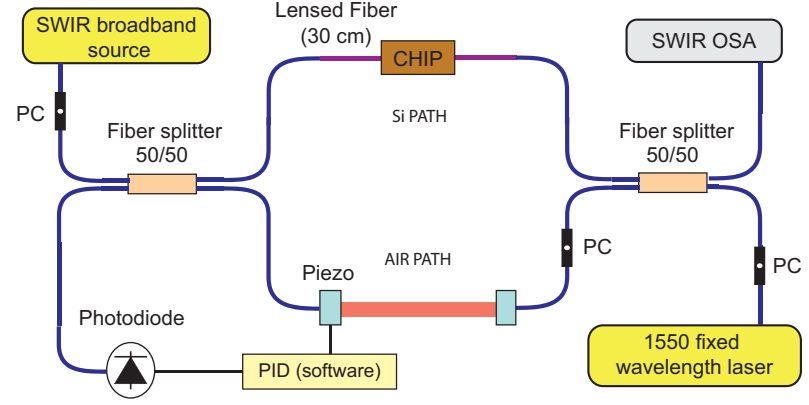

Figure 1. Experimental setup used to measure the chromatic dispersion. It consists of a stabilized Mach-Zehnder interferometer with a tunable air path in one arm and a silicon waveguide in the other. SWIR: short-wave infared, OSA: Optical Spectrum Analyzer, PC: Polarization controller. PD: Photodiode, PID: Proportional-Integral-Derivative Controller. As a broadband source an IPG $\mathrm{Cr}^{2+}$ : ZnSe laser and an Advalue Photonics supercontinuum source (AP-SC-MIR) is used. A Yokogawa AQ6375 SWIR spectrum analyzer is used.

creasing the nonlinear parameter of the waveguide. Our measurement setup is depicted in Figure 1. It consists of a Mach-Zehnder interferometer with an air path in one arm and a silicon waveguide in the other. The length of the air arm is chosen so that the group delay is the same in both arms. The fringe spacing of the output interferogram then depends only on higher order dispersion which can be extracted by careful fitting [10]. The interferometer is stabilized by a feedback loop using a single wavelength $1550 \mathrm{~nm}$ laser and a piezo controller mounted on one of the collimators of the air arm. We use two different light sources : The CW Amplified Spontaneous Emission (ASE) from a $\mathrm{Cr}^{2+}$ : ZnSe laser that extends from $2050 \mathrm{~nm}$ to $2400 \mathrm{~nm}$, and a nanosecond pulsed supercontinuum source that extends from $1950 \mathrm{~nm}$ to $2350 \mathrm{~nm}$. The coupling to the silicon waveguide is performed using lensed fibers. We use a $2 \mathrm{~cm}$ long, nominally $850 \mathrm{~nm}$ wide silicon waveguide that is adiabatically tapered to a width of $3 \mu \mathrm{m}$ to increase the overlap with the mode of the lensed fibers. This $3 \mu \mathrm{m}$ waveguide is $4 \mathrm{~mm}$ long and exhibits very large normal dispersion $\left(\beta_{2} \approx 3 \mathrm{ps}^{2} / \mathrm{m}\right)$ so the accumulated dispersion is of the same order of magnitude as the one expected from the $2 \mathrm{~cm}$ long waveguide. The total measured dispersion would therefore be very low and hence difficult to extract. In order to circumvent this problem, we measured the dispersion of a waveguide that consists of the tapers only and added standard SMF fiber (anomalous in that wavelength region) in order to reduce as much as possible the average group-velocity dispersion of the reference in the spectral region of interest. The experiment is then performed in two steps. First we measure the reference waveguide, that consists of the tapers only. Then we measure our $2 \mathrm{~cm}$ long waveguide and obtain our results by subtracting the total dispersion of both measurements.
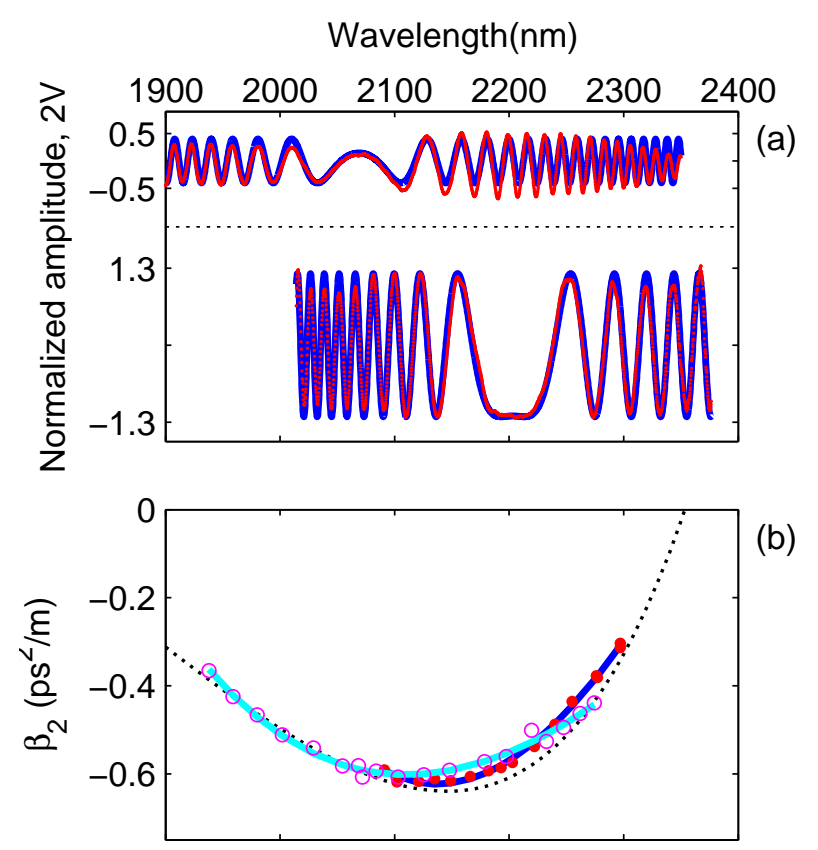

(b)

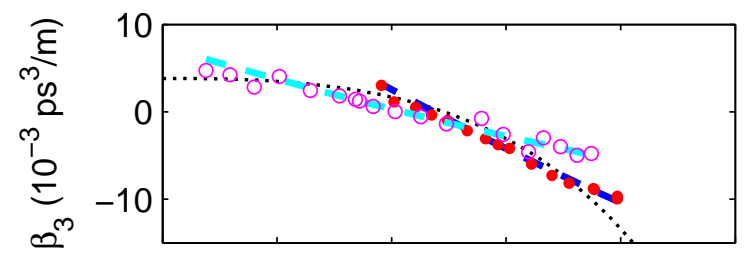

(c)

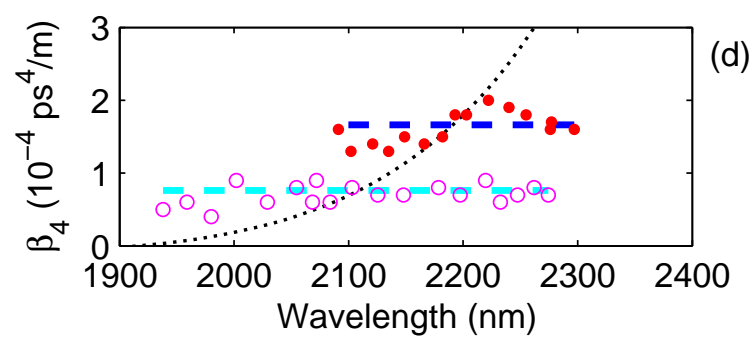

Figure 2. (a) Example of 2 measured interferograms (red) with the pulsed (top) and CW (bottom) sources and corresponding fits (blue) using Eq 4. Experimental data has been smoothed by a Savitsky Golay filter. (b) Extracted SOD profile of our waveguide with the pulsed (circles) and CW (dots) sources. Both are fitted with a second-order polynomial for comparison with extracted higher order dispersion. Experimental results are compared with the second order dispersion (SOD) retrieved from mode solver simulations of a $860 \mathrm{~nm} \mathrm{x}$ $217 \mathrm{~nm}$ (dotted) waveguide. [(c) resp. (d)] Extracted third (resp. fourth) order dispersion with the pulsed (circles) and CW (dots) sources and comparison with first (resp. second) order derivative of the parabolic fit of figure (a) (dashed) and with third order dispersion (resp. fourth order dispersion) retrieved from simulations of a $860 \mathrm{~nm}$ x $217 \mathrm{~nm}$ waveguide (dotted).

In order to extract the dispersion parameters from the interferogram, we mathematically describe it as in [10]

$$
I_{\text {out }}(\omega)=I_{\text {air }}(\omega)+I_{\mathrm{wav}}(\omega)
$$




$$
\begin{aligned}
& +2 V \sqrt{I_{\mathrm{air}}(\omega) I_{\mathrm{wav}}(\omega)} \cos (\phi(\omega)), \\
\phi(\omega)= & \frac{\omega}{c} l_{\mathrm{air}}-\beta(\omega) l_{\mathrm{wav}},
\end{aligned}
$$

where $l_{\text {air }}$ et $l_{\text {wav }}$ are the length of the air and waveguide arms and $I_{\text {air }}\left(\right.$ resp. $I_{\text {wav }}$ ) is the spectrum at the output of the interferometer when the waveguide (resp. air) arm is blocked. We consider here a coherence function $V[16]$ that does not depend on the frequency. By introducing the standard Taylor expansion of the dispersion characteristics of the guided mode [6] in Eq 2, we find :

$$
\begin{aligned}
\phi(\Omega)= & \left(\frac{\omega_{0} l_{\text {air }}}{c}-l_{\text {wav }} \beta_{0}\right)+\Omega\left(\frac{l_{\text {air }}}{c}-l_{\text {wav }} \beta_{1}\right) \\
& -l_{\text {wav }}\left(\frac{1}{2} \beta_{2} \Omega^{2}+\frac{1}{6} \beta_{3} \Omega^{3}+\frac{1}{24} \beta_{4} \Omega^{4} \ldots\right),(3)
\end{aligned}
$$

where we introduced the frequency shift $\Omega=\omega-\omega_{0}$. Choosing $\omega_{0}$ such $\frac{1}{c} l_{\text {air }}=l_{\text {wav }} \beta_{1}$, i.e. cancelling the group delay difference between the two arms, the second term from the right hand side cancels and we finally obtain :

$$
\begin{aligned}
I_{\text {out }}(\Omega)= & I_{\text {air }}(\Omega)+I_{\mathrm{wav}}(\Omega) \\
& +2 V \sqrt{I_{\mathrm{air}}(\Omega) I_{\mathrm{wav}}(\Omega)} \cos (\phi(\Omega)) \\
\phi(\Omega)= & C-l_{\mathrm{wav}}\left(\frac{1}{2} \beta_{2} \Omega^{2}+\frac{1}{6} \beta_{3} \Omega^{3}+\cdots\right) .
\end{aligned}
$$

By slightly changing the length of the air path in the experiment, we modify the central frequency $\omega_{0}$ and can extract the corresponding higher order dispersion of our silicon waveguide by fitting experimental data with Eq 4. Our measurements are performed on a $2 \mathrm{~cm}$ long waveguide. The width and height of the silicon waveguide retreived from SEM are $840 \mathrm{~nm}$ and $230 \mathrm{~nm}$ respectively. The dispersion measurements results are shown in Figure 2. Figure 2(a) shows two examples of experimental interferograms obtained with the nanosecond supercontinuum (SC) source (top) and the linearly polarized CW ASE of the Cr2+:ZnSe laser (bottom). The latter shows a a very flat interferogram with high visibility $(>10 \mathrm{~dB})$. The $\mathrm{SC}$ source results show poorer spectral fringes and a stronger spectral dependence of the coherence function $\mathrm{V}$, which is consistent with coherence studies of SC sources [17]. Both are fitted with Eq 2 by truncating the dispersion expansion at the fourth order, as higher order dispersion didn't show improvement in the fitting. Extracted $2^{\text {nd }}$ to $4^{\text {th }}$ order dispersion coefficients for both sources are presented in Figure 2 (b)-(d). We see measurable differences between the results for the two different sources we use. A parabolic fit of both second order dispersion (SOD) profiles confirms the difference in curvature by a factor 2 . The reason for this is unclear but the measurements with the SC source could be affected by the dispersion of the nonlinear parameter and the complex frequency dependence of the coherence function. However, we believe that the good agreement between both sources validate second and third order dispersion measurements with such a pulsed supercontinuum source which allows to

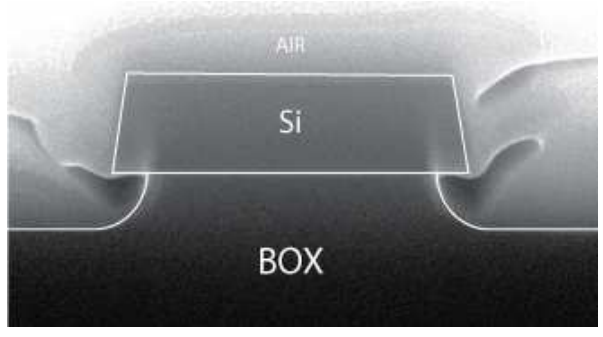

Figure 3. SEM picture of our sample after full underetching with overlay corresponding to the waveguide profile used in simulations. Note that platina was deposited during SEM inspection. Width and height of the simulation profile are chosen to fit the measured SOD profile (see Figure 2 (b)). Radius of the circular undercut value are retrieved from profilometer measurements.

extend our measurement range in the spectral region of thulium-doped fiber sources where CW nonlinear conversion in silicon [18] and supercontinuum generation in amorphous silicon have been demonstrated [19]. Our measurements are compared to simulation results. Using a mode solver, we looked for the best fit of our experimental results within reasonable geometrical variations of the waveguide and found a good reproduction of the measured SOD for a waveguide of $217 \mathrm{~nm} \mathrm{x} 860 \mathrm{~nm}$ (dotted line). There still is however a big discrepancy between simulations and experiment for the fourth order dispersion. This is consistent with previous experimental results of nonlinear conversion in similar waveguides and we refer the reader to [5] for a discussion on possible causes. Our results confirm that the ZDW can be shifted to shorter wavelength from what is expected when designing the waveguide (with a targeted silicon thickness of $220 \mathrm{~nm}$ ). This can be detrimental to nonlinear applications as some sources have limited tunability and working close to the ZDW is essential for applications such as frequency conversion and supercontinuum generation [6]. Moreover, the nonlinear losses of silicon significantly drop in this wavelength range and pushing the anomalous region to longer wavelengths is essential for applications requiring CW pumping where free carrier absorption is detrimental. Tuning the ZDW to longer wavelengths can be achieved by underetching the waveguide, i.e. increasing the vertical confinement. It is performed by wet-etching the waveguide with a solution of diluted $(1 \%)$ HF. Calibration measurements of the underetching with a profilometer showed an etching rate of $1.2 \mathrm{~nm} / \mathrm{min}$. We underetched our waveguides in 2 steps of about $45 \mathrm{~min}$. An etch depth of $55 \mathrm{~nm}$ and $113 \mathrm{~nm}$ was measured. A circular underetching profile was identified by SEM inspection (see Figure 3). We performed a dispersion measurement on the underetched samples after both steps. We used only the CW ASE source as we are mainly interested in the longer wavelength range to show a shift of the ZDW. Results are presented in Figure 4 and compared to simulations of a waveguide with width and height extracted by fitting the SOD curve (before 

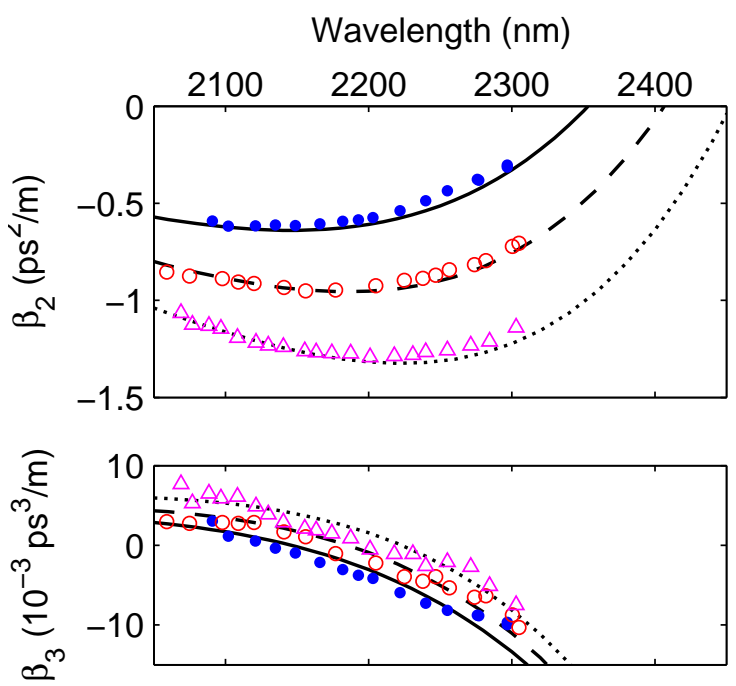

(b)

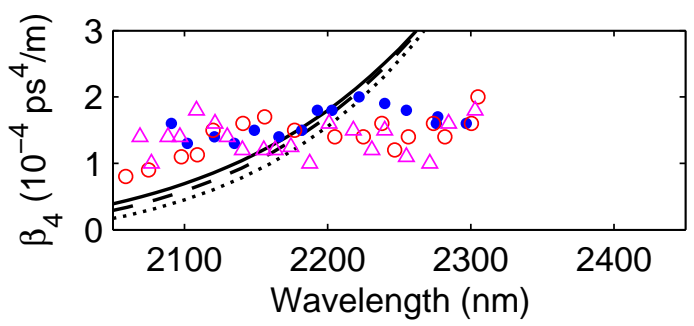

Figure 4. Extracted second (a), third (b) and fourth (c) order dispersion of the waveguides after $0 \mathrm{~nm}$ (dots), $55 \mathrm{~nm}$ (circles) and $113 \mathrm{~nm}$ (triangles) underetching. These results are compared with the corresponding dispersion retrieved from simulations of a $860 \mathrm{nmx} 217 \mathrm{~nm}$ waveguide with 0 (full), 55 (dashed) and 113 (dotted) nm underetching

underetching) and using measured etch depths. An excellent agreement between measured and simulated SOD is found, showing a clear shift of the ZDW after underetching the sample. Very good agreement is also shown for the third-order dispersion. Moreover, both measurement and simulation of the fourth-order dispersion measurements show that the curvature of the SOD profile is left unchanged by the underetching. This confirms the potential of this simple technique for dispersion tuning. The possibility to slowly underetch should allow for very precise tuning of integrated parametric amplifiers. Using a cutback technique we have measured a small increase of propagation losses from $0.6 \mathrm{~dB} / \mathrm{cm}$ before underetching to $0.9 \mathrm{~dB} / \mathrm{cm}$ after $100 \mathrm{~nm}$ of underetching. Moreover, characteristics of high Q microresonators [20] and diffraction gratings for fiber-to-chip coupling for the short-wave infrared [21] were left unchanged when underetched up to $100 \mathrm{~nm}$.

In conclusion we have demonstrated the measurement and tuning of second-to-fourth order dispersion of a silicon wire waveguide from $1950 \mathrm{~nm}$ to $2300 \mathrm{~nm}$. We show good agreement with simulations of a waveguide with width and height within $5 \%$ from the values retrieved by SEM inspection. Dispersion tuning was performed by underetching and excellent agreement of the shift of the SOD profile between measurements and simulations is found. We believe further improvements, mainly reducing the overall loss of the measurement system, will allow to work without tapers and with longer waveguides. This should allow for very precise measurements of higher-order dispersion which are essential to simulations of supercontinuum generation [22].

\section{References}

[1] J. Leuthold, C. Koos and W. Freude, Nat Photon 4, 535544 (2010).

[2] H. K. Tsang, C. S. Wong, T. K. Liang, I. E. Day, S. W. Roberts, A. Harpin, J. Drake, and M. Asghari, Applied Physics Letters 80, 416 (2002).

[3] X. Liu, J. B. Driscoll, J. I. Dadap, R. M. Osgood, S. Assefa, Y. A. Vlasov, and W. M. J. Green, Opt. Express 19, 7778 (2011).

[4] X. Liu, R. M. Osgood, Y. A. Vlasov, and W. M. J. Green, Nature Photonics 4, 557(2010).

[5] B. Kuyken, X. Liu, R. M. Osgood Jr., R. Baets, G. Roelkens, and W. M. J. Green, Opt. Express 19, 20172 (2011).

[6] G. P. Agrawal, Nonlinear Fiber Optics, 4th ed. ,Academic Press (2006).

[7] A. C. Turner, C. Manolatou, B. S. Schmidt, M. Lipson, M. A. Foster, J. E. Sharping, and A. L. Gaeta, Opt. Express 14, 4357 (2006).

[8] http://www.epixfab.eu

[9] B. Kuyken, X. Liu, G. Roelkens, R. Baets, J. Osgood, and W. M. J. Green, Opt. Lett. 36, 4401 (2011).

[10] P. A. Merritt, R. P. Tatam, and D. A. Jackson, Journal of Lightwave Technology 7, 703 (1989).

[11] D. W. Kim, S. H. Kim, S. H. Lee, K.-H. Kim, J.-M. Lee, and E.-H. Lee, Journal of Lightwave Technology 30, 43 (2012).

[12] S. Mas, J. Matres, J. Marti, and C. J. Oton, IEEE Photonics Journal 4, 825 (2012).

[13] E. Dulkeith, F. Xia, L. Schares, W. M. J. Green, and Y. A. Vlasov, Opt. Express 14, 3853 (2006).

[14] X. Liu, W. M. J. Green, X. Chen, I.-W. Hsieh, J. I. Dadap, Y. A. Vlasov, and J. Osgood, Opt. Lett. 33, 2889 (2008).

[15] J. Riemensberger, K. Hartinger, T. Herr, V. Brasch, R. Holzwarth, and T. J. Kippenberg, Opt. Express 20, 27661 (2012).

[16] E. Hecht and A. Zajac, Optics, Addison-Wesley 2 (2002)

[17] J.M. Dudley and S. Coen, Opt. Lett. 27, 1180 (2002).

[18] R. K. W. Lau, M. Mnard, Y. Okawachi, M. A. Foster, A. C. Turner-Foster, R. Salem, M. Lipson, and A. L. Gaeta, Opt. Lett. 36, 1263 (2011).

[19] U. Dave, S.Uvin, B. Kuyken, S. Selvaraja, F. Leo, G. Roelkens, Submitted.

[20] F. Leo, B. Kuyken, N. Hattasan, R. Baets and G. Roelkens, European Conference on Integrated Optics (ECIO 2012), 156158, Spain (2012)

[21] N. Hattasan, B. Kuyken, F. Leo, E. M. P. Ryckeboer, D. Vermeulen, and G. Roelkens, IEEE Photonics Technology Letters 24, 1536(2012).

[22] L. Yin, Q. Lin and G.P. Agrawal, Opt. Lett. 32, 391 (2007). 


\section{References}

[1] J. Leuthold, C. Koos and W. Freude, Nonlinear silicon photonics, Nat Photon 4, 535544 (2010).

[2] H. K. Tsang, C. S. Wong, T. K. Liang, I. E. Day, S. W. Roberts, A. Harpin, J. Drake, and M. Asghari, Optical dispersion, two-photon absorption and self-phase modulation in silicon waveguides at $1.5 \mu \mathrm{m}$ wavelength, Applied Physics Letters 80, 416 (2002).

[3] X. Liu, J. B. Driscoll, J. I. Dadap, R. M. Osgood, S. Assefa, Y. A. Vlasov, and W. M. J. Green, Self-phase modulation and nonlinear loss in silicon nanophotonic wires near the mid-infrared two-photon absorption edge, Opt. Express 19, 7778 (2011).

[4] X. Liu, R. M. Osgood, Y. A. Vlasov, and W. M. J. Green, Mid-infrared optical parametric amplifier using silicon nanophotonic waveguides, Nature Photonics 4, $557(2010)$

[5] B. Kuyken, X. Liu, R. M. Osgood Jr., R. Baets, G. Roelkens, and W. M. J. Green, Mid-infrared to telecomband supercontinuum generation in highly nonlinear silicon-on-insulator wire waveguides, Opt. Express 19, 20172 (2011).

[6] G. P. Agrawal, Nonlinear Fiber Optics, 4th ed. ,Academic Press (2006).

[7] A. C. Turner, C. Manolatou, B. S. Schmidt, M. Lipson, M. A. Foster, J. E. Sharping, and A. L. Gaeta, Tailored anomalous group-velocity dispersion in silicon channel waveguides, Opt. Express 14, 4357 (2006).

[8] http://www.epixfab.eu

[9] B. Kuyken, X. Liu, G. Roelkens, R. Baets, J. Osgood, and W. M. J. Green, $50 \mathrm{~dB}$ parametric on-chip gain in silicon photonic wires, Opt. Lett. 36, 4401 (2011).

[10] P. A. Merritt, R. P. Tatam, and D. A. Jackson, Interferometric chromatic dispersion measurements on short lengths of monomode optical fiber, Journal of Lightwave Technology 7, 703 (1989).

[11] D. W. Kim, S. H. Kim, S. H. Lee, K.-H. Kim, J.-M. Lee, and E.-H. Lee, A New Method of Measuring Localized Chromatic Dispersion of Structured Nanowaveguide Devices Using White-Light Interferometry, Journal of Lightwave Technology 30, 43 (2012).
[12] S. Mas, J. Matres, J. Marti, and C. J. Oton, Accurate Chromatic Dispersion Characterization of Photonic Integrated Circuits, IEEE Photonics Journal 4, 825 (2012).

[13] E. Dulkeith, F. Xia, L. Schares, W. M. J. Green, and Y. A. Vlasov, Group index and group velocity dispersion in silicon-on-insulator photonic wires, Opt. Express 14, 3853 (2006).

[14] X. Liu, W. M. J. Green, X. Chen, I.-W. Hsieh, J. I. Dadap, Y. A. Vlasov, and J. Osgood, Conformal dielectric overlayers for engineering dispersion and effective nonlinearity of silicon nanophotonic wires, Opt. Lett. 33, 2889 (2008).

[15] J. Riemensberger, K. Hartinger, T. Herr, V. Brasch, R. Holzwarth, and T. J. Kippenberg, Dispersion engineering of thick high-Q silicon nitride ring-resonators via atomic layer deposition, Opt. Express 20, 27661 (2012).

[16] E. Hechtand A. Zajac, Optics, Addison-Wesley 2 (2002)

[17] J.M. Dudley and S. Coen, Coherence properties of supercontinuum spectra generated in photonic crystal and tapered optical fibers, Opt. Lett. 27, 1180 (2002).

[18] R. K. W. Lau, M. Mnard, Y. Okawachi, M. A. Foster, A. C. Turner-Foster, R. Salem, M. Lipson, and A. L. Gaeta, Continuous-wave mid-infrared frequency conversion in silicon nanowaveguides, Opt. Lett. 36, 1263 (2011).

[19] U. Dave, S.Uvin, B. Kuyken, S. Selvaraja, F. Leo, G. Roelkens, Telecom to mid-infrared spanning supercontinuum generation in hydrogenated amorphous silicon waveguides, Submitted.

[20] F. Leo, B. Kuyken, N. Hattasan, R. Baets and G. Roelkens, Passive SOI devices for the short-wave infrared, European Conference on Integrated Optics (ECIO 2012), 156158, Spain (2012)

[21] N. Hattasan, B. Kuyken, F. Leo, E. M. P. Ryckeboer, D. Vermeulen, and G. Roelkens, High-Efficiency SOI Fiberto-Chip Grating Couplers and Low-Loss Waveguides for the Short-Wave Infrared, IEEE Photonics Technology Letters 24, 1536 (2012).

[22] L. Yin, Q. Lin and G.P. Agrawal, Soliton fission and supercontinuum generation in silicon waveguides, Opt. Lett. 32, 391(2007). 